\title{
Rendimiento del jugador de baloncesto en silla de ruedas según la estadística de juego
}

\section{Wheelchair basketball player performance by game statistics}

\section{Desempenho do jogador de basquetebol na cadeira de rodas por estatísticas de jogo}

\author{
Javier Pérez-Tejero ${ }^{1,2}$ y Javier Pinilla Arbex ${ }^{1,2}$ \\ 1 Universidad Politécnica de Madrid - Centro de Estudios Sobre Deporte Inclusivo y 2 Federación Española de Deportes para Personas con Discapacidad Física
}

Resumen: El análisis del rendimiento deportivo del jugador de baloncesto en silla de ruedas (BSR) ha sido un creciente objeto estudio en los últimos años. Sin embargo, escasos estudios han incidido en las estadísticas de juego. Por ello, el presente estudio tiene por objeto determinar un referente de rendimiento máximo para cada clase funcional en base a cada variable de la estadística de juego propia de la competición. Para ello, se obtuvieron las estadísticas de juego oficiales de los 32 partidos disputados por los 8 mejores equipos de España en la fase por el título de la División de Honor de BSR (temporada 2013/2014), recabando información por jugador y partido; en total fueron estudiados 58 jugadores correspondientes a la clases $1(n=11)$, $2(n=12), 3(n=12)$ y $4(n=23)$. En relación a los porcentajes de tiro, el de $2 p$ sitúa entre el 60,8-67,6\% de acierto, realizando más lanzamientos a mayor clase funcional. Para los lanzamientos de 3p fue de 41,2-42,9\% (sólo clases 2,3 y 4). Para los lanzamientos de 1 p fue de 66,7\%-89,5\%) siendo el jugador clase 4 el porcentaje mayor y más número de lanzamientos realizados. Los datos máximos obtenidos se registraron en los jugadores de clase 4, con más rebotes ofensivos $(4,12)$ y defensivos $(9,99)$ registrados, así como faltas recibidas $(9,95)$, asistencias $(10,8)$, robos $(1,99)$ y puntos anotados $(29,4)$. Este estudio ha permitido la caracterización del rendimiento en este deporte, destacando la importancia contrastar el rendimiento de cada jugador con los de su misma clase funcional según la estadística de juego.

Palabras clave: Baloncesto en silla de ruedas, clasificación funcional, estadísticas de juego, rendimiento deportivo.

Abstract: The analysis of the sport performance in wheelchair basketball (WB) has been a growing topic in recent years. However, few studies have focused on the game statistics. Therefore, the present study is to determine a reference for maximum performance for each functional class based on each variable from game related statistics. To this end, official statistics from 32 matches for the top 8 teams in the title league at the Spanish Honour Division (season 2013/2014) were obtained with information per player and game; a total of 58 players corresponding to the classes $1(n=11), 2(n=12), 3$ $(\mathrm{n}=12)$ and $4(\mathrm{n}=23)$ were studied. Regarding the shooting percentages, the 2-points was 60.8 to $67.6 \%$ accuracy, making more shots in higher functional classes. 3-points was 41.2 to $42.9 \%$ (only classes 2,3 and 4 ). 1-point free throw was $66.7 \%-89.5 \%$, with class 4 player with the highest percentage and number of throws made. The maximum data obtained were recorded in class 4 players with more offensive rebounds (4.12) and defensive (9.99) registered and fouls received (9.95), assists (10.8), steals (1.99) and points scored (29.4). This study has allowed the characterization of performance in WB, stressing the importance in comparing the performance of each player with those of the same functional class per game statistics.

Keywords: wheelchair basketball, functional classification, game related statistics, sports performance.

Resumo: A análise do desempenho esportivo jogador de basquete em cadeira de rodas (BSR) tem sido um objeto de estudo crescente nos últimos anos. Entretanto, poucos estudos têm-se centrado nas estatísticas do jogo. Portanto, o presente estudo é determinar um valor de referência para o desempenho máximo para cada classe funcional com base em cada estatística próprio jogo variável da competição. Para este fim, as estatísticas oficiais jogar 32 partidas para o top 8 equipas na fase de Espanha para o título da Divisão de Honra da BSR (2013/2014 temporada) foram obtidos mediante a obtenção de informaçôes por jogador e caça; Foram estudados 58 jogadores totais correspondentes aos ramos $1(n=11), 2(n=12), 3(n=12)$ e $4(n=23)$. Em relação às percentagens de disparo, o ponto 2 é a precisão entre $60,8-67,6 \%$, tornando mais lotes para a classe funcional maior. 3 p para lançamentos foi 41,2-42,9\% (apenas as classes de 2,3 e 4). $1 \mathrm{p}$ de lançamentos foi de 66,7\% -89,5\%) Classe 4 jogador com a percentagem eo número de lances feitos mais alto. Os dados máximos obtidos foram registrados na classe 4 jogadores com mais rebotes ofensivos (4.12) e defensiva $(9,99)$ registrado e faltas recebidas $(9,95)$, assistências $(10,8)$, rouba $(1,99)$ e pontos marcados $(29,4)$. Este estudo permitiu a caracterizaçáo do desempenho no esporte, salientando a importância de comparar o desempenho de cada jogador com os da mesma classe funcional como estatísticas de jogo. Palavras-chave: basquetebol de cadeira de rodas, classificação funcional, estatísticas de jogo, desempenho esportivo.

\section{Introducción}

Tradicionalmente y también en la actualidad, la investigación ha orientado su interés en el análisis de la clasificación fun-

Dirección para correspondencia [Correspondence address]: Javier Perez Tejero. Departamento de Salud y Rendimiento Humano. Edificio Central, 3a Planta, Despacho CEDI. Buzón: 43. Universidad Politécnica de Madrid (UPM).E-mail: j.perez@upm.es cional (De Groot, Balvers, Kouwenhoven, y Janssen, 2012; Tweedy y Vanlandewijck, 2011; Vanlandewijck, Verellen, y Tweedy, 2011) y su impacto sobre el rendimiento en el baloncesto en sillas de ruedas (BSR). Estos estudios, han permitido ampliar sustancialmente el conocimiento sobre la importancia de la posición del jugador en la silla de ruedas (Mason, 
Woude, y Goosey-Tolfrey, 2013; Vanlandewijck et al., 2011) y el rendimiento físico de acuerdo a la clase funcional (de Lira et al., 2010; Molik, Kosmol, Morgulec, Hübner-Woźniak, y Rutkowska, 2006; Pérez, 2003). Sin embargo, el interés por el análisis del rendimiento de la propia competición, como sugiere Nadori (1993), para estudiar el rendimiento real del deportista, está siendo una línea de investigación reciente para conocer el rendimiento del jugador de BSR de alta competición.

De acuerdo al análisis de las estadísticas de juego, Gómez, Pérez, Molik, Szyman, y Sampaio (2014) identificaron los lanzamientos de 2 puntos encestados, los tiros libres lanzados y fallados, canastas de 3 puntos falladas, asistencias y faltas recibidas como variables discriminantes entre ganadores y perdedores en los partidos igualados en el BSR masculino, mientras que para los partido igualados femeninos, solo los lanzamientos de 2 puntos encestados discriminaron entre ganadoras y perdedoras. Vanlandewijck et al. (2004), dentro también de la competición femenina, identificaron que las jugadoras de la clase 1 realizaban menos asistencias, las de la clase 3 realizaban un mayor número de bloqueos ciegos y las de la clase 4 forzaban más fallos, realizaba más tapones, lanzaban más tiros libres y canastas de dos puntos. Acorde a estos resultados, los datos del estudio de Skučas, Stonkus, Molik, y Skučas (2009) en BSR masculino registraron un mayor número de acciones de los jugadores de la Clase 4 frente a las demás clases. Estas diferencias en la clase funcional también fueron detectadas por Molik et al. (2009), afirmando además la existencia de más semejanzas en el rendimiento entre clases funcionales próximas y atribuyendo el éxito a los equipos con más eficacia en el lanzamiento.

En esta línea, el presente estudio tiene por objeto analizar el rendimiento durante la máxima competición española de BSR (División de Honor) a partir de la estadística de juego (Gomez, Lorenzo, Sampaio, Ibanez, y Ortega, 2008) y caracterizar el rendimiento individual del jugador de acuerdo a su clase funcional, con el fin de que pueda servir como referente en la planificación del entrenamiento y el establecimiento de objetivos para los jugadores de BSR de alta competición.

\section{Método}

Se analizó el rendimiento de los jugadores de baloncesto en silla de ruedas de la máxima competición española en este deporte (División de Honor) durante la fase por el título en la temporada 2013/2014, en la cual participaron los 8 mejores equipos de la liga. Para ello, se obtuvieron las estadísticas de juego oficiales (FIBA, 2005), facilitadas por la Federación Española de Deportes para Personas con Discapacidad Física (FEDDF), correspondientes a los 32 partidos durante esta fase en la cual participaron 94 jugadores.

Con el fin de garantizar el análisis del rendimiento de aquellos jugadores cuya participación fue más representativa durante la competición, se seleccionaron los datos estadísticos de aquellos jugadores que habían jugado más de 10 minutos de media por partido (Gómez y Lorenzo, 2007), siendo un total de 58 jugadores que correspondían a la Clase $1(\mathrm{n}=11)$, Clase $2(n=12)$, Clase $3(n=12)$ y Clase $4(n=23)$.

Los datos fueron transcritos a una hoja de cálculo (Excell 2010) y se computó, para las variables de la estadística de juego de cada jugador, el dato acumulado en cada variable durante los 8 partidos jugados. Posteriormente se relativizaron los datos totales obtenidos por cada jugador a 40 minutos de juego, de acuerdo con Vanlandewijck et al. (2004), con el fin de reducir el impacto de la diferencia de minutos jugados por los jugadores y así conocer los resultados obtenidos por cada jugador por cada 40 minutos jugados (un partido completo). Este cálculo se llevó a cabo bajo la siguiente fórmula:

$$
\frac{\text { Datos acumulados en } 8 \text { partidos }}{\text { Minutos jugados en } 8 \text { partidos }} \times 40
$$

A partir de estos datos, se realizó un análisis descriptivo del rendimiento de los jugadores por cada clase funcional estableciendo los valores medios y desviación típica. Se aplicó la prueba de Kolmogorov-Smirnoff para estudiar la normalidad de la distribución de los datos, que resultó positiva, por lo que se utilizó estadística paramétrica. Se analizaron las diferencias entre clases funcionales usando el test U Mann Whitney, así como la correlación de Pearson para estudiar la relación entre las clases funciones y las variables de las estadísticas de juego. Así mismo, se detectaron los mejores valores registrados en cada variable de juego para cada clase funcional con el fin de determinar el rendimiento óptimo del jugador de BSR por cada clase. El análisis estadístico se llevó a cabo a través del programa Excel 2010 y el paquete estadístico SPSS 20.0.

\section{Resultados}

Como resultado del análisis de la estadística de juego, en la Tabla 1 se han muestran los datos descriptivos medios para cada variable de juego y clase funcional relativos a cada 40 minutos jugados. La variable "minutos jugados" es la única que no está relativizada ya que muestra los minutos jugados medios por partido. 
Tabla 1. Rendimiento medio por cada clase funcional relativo a 40 minutos de juego durante la fase por el título.

\begin{tabular}{|c|c|c|c|c|c|c|c|c|c|c|c|c|}
\hline \multirow{2}{*}{ VARIABLES } & \multicolumn{3}{|c|}{ Clase 1} & \multicolumn{3}{|c|}{ Clase 2} & \multicolumn{3}{|c|}{ Clase 3} & \multicolumn{3}{|c|}{ Clase 4} \\
\hline & $\mathrm{X}$ & DT & df & $\mathrm{X}$ & DT & df & $\mathrm{X}$ & DT & $\mathrm{df}$ & $\mathrm{X}$ & DT & $\mathrm{df}$ \\
\hline Minutos jugados & 22,93 & 8,8 & & 25,74 & 7,7 & & 23,26 & 8,0 & & 25,10 & 6,4 & \\
\hline 2 p lanzados & 5,52 & 5,4 & $2,3,4$ & 13,62 & 6,2 & 1,4 & 16,53 & 6,0 & 1,4 & 23,77 & 8,1 & $1,2,3$ \\
\hline Porcentaje $2 \mathrm{p}$ & 44,0 & 34,0 & & 31,86 & 10,3 & & 40,1 & 21,6 & & 36,3 & 10,8 & \\
\hline 3 p lanzados & 0,0 & 0,0 & 2,4 & 1,85 & 2,5 & 1 & 1,38 & 2,5 & & 2,93 & 4,5 & 1 \\
\hline Porcentaje $3 p$ & -- & -- & & 19,9 & 17,5 & & 29,85 & 16,1 & & 28,51 & 14,0 & \\
\hline Tiros libres lanzados & 0,69 & 0,7 & $2,3,4$ & 3,39 & 3,0 & 1,4 & 4,53 & 2,4 & 1 & 6,16 & 3,4 & 1,2 \\
\hline Porcentaje tiros libres & 47,61 & 39,0 & & 43,75 & 14,0 & 4 & 52,29 & 20,4 & & 58,63 & 16,8 & 2 \\
\hline Rebotes ofensivos & 1,29 & 0,9 & $2,3,4$ & 2,05 & 1,3 & 1 & 2,55 & 1,4 & 1,4 & 2,96 & 1,3 & 1,3 \\
\hline Rebotes defensivos & 2,1 & 1,5 & $2,3,4$ & 4,5 & 2,5 & 1,4 & 6,15 & 3,6 & 1,4 & 11,1 & 4,0 & $1,2,3$ \\
\hline Asistencias & 1,29 & 1,36 & $2,3,4$ & 4,62 & 2,9 & 1 & 4,5 & 3,0 & 1 & 6,08 & 4,6 & 1 \\
\hline Faltas cometidas & 3,81 & 1,8 & 2 & 5,15 & 2,1 & 1 & 4,5 & 2,0 & & 5,2 & 2,1 & \\
\hline Faltas recibidas & 1,26 & 0,7 & 3,4 & 3,58 & 2,9 & & 4,32 & 3,0 & 1 & 5,13 & 3,4 & 1 \\
\hline Pérdidas & 1,68 & 1,0 & 2,4 & 3,32 & 1,7 & 1 & 3,03 & 1,5 & & 4,06 & 2,2 & 1 \\
\hline Robos & 0,81 & 0,5 & 4 & 1,08 & 1,0 & & 1,08 & 0,7 & & 1,65 & 1,0 & 1 \\
\hline Tapones cometidos & 0,18 & 0,5 & & 0,05 & 0,1 & 4 & 0,21 & 0,5 & & 0,29 & 0,4 & 2 \\
\hline Puntos & 5,4 & 5,2 & $2,3,4$ & 15,21 & 8,7 & 1,4 & 18,99 & 7,8 & 1,4 & 30,95 & 12,3 & $1,2,3$ \\
\hline
\end{tabular}

*df (hace referencia a las clases con las que existen diferencias significativas, $\mathrm{p} \leq 0,05$ )

El análisis en el rendimiento de las clases funcionales no mostró diferencias significativas $(\mathrm{p} \leq 0,05)$ en cuanto a los minutos medios jugados $(24,5 \pm 7,4)$ el porcentaje de lanzamiento de canastas de 2 puntos, 3 puntos y tiros libres, a excepción de la diferencia entre las clases 2 y 4 en esta última variable. Sin embargo, resaltan las diferencias que se registraron entre la clase 1 y las otras tres clases para las variables: número de lanzamientos de 2 puntos, tiros libres lanzados, rebotes ofensivos y defensivos, asistencias y puntos; variables en las que los jugadores de la Clase 1 obtienen valores inferiores respecto de las demás clases. Así mismo, para la Clase 1 no se registró ningún lanzamiento de 3 puntos durante toda la fase por el título, recibieron menos faltas que las Clases 3 y 4 , pero cometieron menos faltas que la Clase 2 y menos pérdidas de balón que las Clases 2 y 4 .

Al contrario que la Clase 1, la Clase 4 mostró valores significativamente superiores $(p \leq 0,05)$ que las otras tres clases en las variables de lanzamientos de 2 puntos realizados, rebotes defensivos obtenidos y puntos marcados. También esta Clase 4 mostró valores superiores que las Clases 1 y 2 en el número de tiros libres lanzados y que las Clases 1 y 3 el en número de rebotes ofensivos. En relación a las Clases 2 y 3, éstas no mostraron diferencias significativas entre sí para ninguna variable y se situaron en una posición intermedia significativamente respecto de las clases 1 y 4 , para las variables de lanzamientos de 2 puntos realizados, rebotes defensivos y puntos anotados.

En la Tabla 2 se muestran los mejores valores registrados para cada clase funcional en cada variable de juego, no correspondiendo todos los datos a un mismo jugador sino al mejor resultado obtenido durante los 8 partidos para una misma clase funcional en esa variable. Estos datos demuestran que el mejor rendimiento encontrado para todas las clases en relación al porcentaje de lanzamiento de 2 puntos oscila entre el 60,8 (Clase 2) y el 67,6\% (Clase 4), sin embargo, aunque el porcentaje de acierto es próximo entre clases funcionales, a mayor clase funcional, mayor es el número de lanzamientos realizados durante el mismo tiempo de juego. De manera similar ocurre en los lanzamientos de 3 puntos, en los que el porcentaje de lanzamiento es cercano ( 41,2 a $42,9 \%$ ), pero el número de lanzamientos asciende a medida que lo hace la clase funcional.

Esta línea ascendente en la que el rendimiento máximo obtenido por un jugador es mayor a medida que aumenta la clase funcional, también se reproduce para el número de rebotes defensivos logrados, faltas recibidas y puntos anotados. Los lanzamientos de 1p son los que cuentan con mayor porcentaje de acierto de entre todos los lanzamientos, situándose este porcentaje entre el $66,7 \%$ y el $89,5 \%$. El jugador de la Clase 4 es el que cuenta con mayor acierto y número de lanzamientos realizados y a su vez también obtuvo en cada variable los mejores resultados en torno a rebotes ofensivos $(4,12)$ y defensivos $(9,99)$, menor número de pérdidas $(0,31)$, más faltas recibidas $(9,95)$, asistencias $(10,8)$, robos $(1,99)$ y puntos anotados $(29,4)$. 
Tabla 2. Máximas variables de rendimiento para cada clase funcional relativos a 40 minutos de juego.

\begin{tabular}{|c|c|c|c|c|c|c|c|c|c|c|c|c|c|c|}
\hline & \multicolumn{2}{|c|}{ Tiros de $2 \mathrm{p}$} & \multicolumn{2}{|c|}{ Tiros de $3 p$} & \multicolumn{2}{|c|}{ Tiros de $1 \mathrm{p}$} & \multicolumn{2}{|c|}{ Rebotes } & \multicolumn{2}{|c|}{ Faltas } & \multirow[b]{2}{*}{ Asist. } & \multirow[b]{2}{*}{ Pérd. } & \multirow[b]{2}{*}{ Robos } & \multirow[b]{2}{*}{ Pts. } \\
\hline & Lanz. & $\%$ & Lanz. & $\%$ & Lanz. & $\%$ & Of. & Def. & Com. & Rec. & & & & \\
\hline Clase 1 & 6,35 & 61,5 & 0 & - & 1,27 & 83,3 & 2,12 & 3,38 & 0,8 & 1,41 & 3,91 & 0,42 & 1,96 & 7,81 \\
\hline Clase 2 & 9,76 & 60,8 & 1,08 & 42,9 & 3,48 & 66,7 & 2,1 & 4,23 & 1,56 & 4,81 & 6,24 & 0,8 & 1,95 & 16,8 \\
\hline Clase 3 & 11,3 & 64,8 & 2,63 & 41,2 & 2,32 & 66,7 & 2,76 & 7,06 & 1,37 & 5,65 & 5,52 & 1,07 & 1,47 & 17,5 \\
\hline Clase 4 & 13,5 & 67,6 & 7,84 & 41,4 & 3,96 & 89,5 & 4,12 & 9,99 & 1,77 & 9,95 & 10,8 & 0,31 & 1,99 & 29,4 \\
\hline
\end{tabular}

El análisis de las correlaciones entre la clase funcional y las variables de juego corroboraron la existencia de una estrecha relación $(\mathrm{p} \leq 0,05)$ positiva entre la clase funcional y los resultados de las variables de juego: número de lanzamientos de 2 puntos $(\mathrm{r}=0,71)$, canastas de 2 puntos encestadas $(\mathrm{r}=0,78)$, tiros libres lanzados $(r=0,62)$, tiros libres encestados $(r=0,66)$, rebotes defensivos $(r=0,79)$, y puntos anotados $(r=0,73)$. Aunque múltiples variables mostraron correlaciones positivas entre sí, al analizar la correlación parcial de las variables controlando la clase funcional, no se reflejaron correlaciones significativas entre ninguna variable por lo que la variable clase funcional resultó ser la determinante de estas correlaciones.

\section{Discusión}

Es destacable la originalidad del estudio presentado, tanto por la actualidad y el nivel de rendimiento de los datos obtenidos, como la caracterización de cada clase y variable. Al describirse los niveles de rendimiento individuales medios y máximos del jugador de BSR de la División de Honor española de acuerdo a su clasificación funcional. Destaca en la muestra de este análisis, la presencia de cerca del doble de jugadores de la Clase 4 ( $n=23)$ que de las demás clases (Clase $1 \mathrm{n}=11$, Clase $2 \mathrm{n}=12$ y Clase $3 \mathrm{n}=12$ ), lo cual representa la prevalencia de la rotación 1-2-3-4-4 como quinteto principal en pista en esta competición frente a otras posibles rotaciones como 1-1-4-4-4 (juego reforzado sobre las puntuaciones altas) o bien 1-3-3-3-4 (juego reforzado sobre las puntuaciones intermedias). La presencia de dos hombres altos parece tomar relevancia en este baloncesto frente al baloncesto convencional en la que se tiende a jugar con 4 jugadores exteriores y un interior (Sampaio et al., 2009).

La presencia de la clasificación funcional, la cual otorga mayor puntuación a los jugadores con mayor movilidad (IWBF, 2010), parece estar muy presente en el rendimiento en este deporte en la línea de estudios anteriores (Gómez, Pérez, Molik, Szyman, y Sampaio, 2014; Vanlandewijck et al., 2004), enfatizando la labor de los jugadores de la Clase 4 ya que se diferencian significativamente de las otras clases en el número de lanzamientos de dos puntos, rebotes defensivos y puntos encestados. La posición de estos jugadores en la silla de ruedas suele ser más elevada debido a un mayor control del tronco que en las clases inferiores (Pérez y Sampedro, 2003) y les permite por lo tanto tener más opciones de obtener un rebote o hacer un lanzamiento a canasta más cercano al aro y con más dificultades de los oponentes de defender dicho lanzamiento (Gordon, 2010); factores que pueden contribuir a la existencia de estas diferencias en los resultados.

Este rol de juego del jugador Clase 4 en el entorno cercano a la canasta se ve reflejada en la estadística a través de las faltas recibidas y cometidas $(5,13$ y 5,20 faltas por cada 40 minutos de juego respectivamente) respecto de la Clase 1 y en el número de tiros libres lanzados respecto de las Clases 1 y 2. Estos valores sugieren la necesidad de rotar a los jugadores Clase 4 para preservarles durante el partido de cometer la quinta falta y debido a la alta exigencia física a la que se ven sometidos; este último aspecto se ve también reflejado en el baloncesto convencional (Gómez y Lorenzo, 2007; Sampaio, Janeira, Ibanez, y Lorenzo, 2006).

En la Clase 1 también se ve reflejado la importancia de la clasificación funcional en el rendimiento (Vanlandewijck et al., 2004), al mostrar valores significativamente inferiores a las demás clases en las variables de lanzamientos de dos puntos, tiros libres lanzados, rebotes ofensivos y defensivos, asistencias y puntos. Esto no significa de ningún modo que el rendimiento de los jugadores de Clase 1 sea inferior, sino que de acuerdo a su capacidad de movimiento y rendimiento físico (de Lira et al., 2010), su posición en la silla (Crespo-Ruiz, Del Ama-Espinosa, y Gil-Agudo, 2011; Vanlandewijck et al., 2011) y su ubicación en el terreno de juego (Vanlandewijck et al., 2004); la labor de las acciones de los jugadores de Clase 1 parecen estar más relacionadas con el desarrollo de acciones colectivas como bloqueos, pantallas, generar desigualdades numéricas de ventaja (man out) o cambios defensivos con ventaja (missmatch) que favorecen el desarrollo de acciones ofensivas de los compañeros. Estas acciones, que no aparecen reflejadas en la estadística de juego, pueden contribuir a que su labor se vea reflejada en las acciones de los compañeros y de los puntos altos; no obstante, la suma de todas las aportaciones de todas los jugadores son las que finalmente discriminan a los equipos ganadores de los perdedores (Gómez et al., 2014).

A la vista de estas diferencias, parece importante que el 
rendimiento del jugador en pista sea contrastado con los valores de rendimiento de los jugadores de la misma clase funcional y de acuerdo a la posición de juego, en la que según Vanlandewijck et al. (2004), el 83\% de los jugadores de la Clase I jugaban como bases, la Clase II y III como aleros (70\%) y la clase 4 como pívots (93\%). Es más, podemos concluir que la clasificación funcional tiene una influencia crucial en el rol del jugador en pista (Pérez, 2012).

Los jugadores de las clases 2 y 3, de acuerdo al sistema de clasificación (IWBF, 2010) son jugadores con un grado de movilidad intermedia entre los jugadores de la Clase $1 \mathrm{y}$ Clase 4, reflejándose este hecho en la configuración de la silla de ruedas (Mason et al., 2013; Vanlandewijck et al., 2004), en el rendimiento físico (de Lira et al., 2010; Leicht, Bishop, y Goosey-Tolfrey, 2012) y la posición ocupada en el terreno de juego (Vanlandewijck et al. 2004). Estas condiciones se ven reflejadas directamente sobre la estadística de juego, en la que los jugadores de las clases 2 y 3 mostraron valores intermedios significativamente diferentes $(\mathrm{p} \leq 0,05)$ a las Clases 1 y 4 en el número de lanzamientos de 2 puntos realizados, rebotes defensivos y puntos anotados.

Estas diferencias, junto a las correlaciones significativas $(\mathrm{p} \leq 0,05)$ existentes entre la clase funcional y el número de lanzamientos de 2 puntos, canastas de 2 puntos encestadas, tiros libres lanzados, tiros libres encestados, rebotes defensivos y puntos anotados reflejan que a medida que incrementa la clase funcional, la implicación directa del jugador con las acciones que se realizan en torno al aro, y que se reflejan en la estadística oficial de juego (FIBA, 2005), parecen ser mayores. Este hecho refleja una importante diferencia respecto al baloncesto convencional y es que, mientras las acciones ofensivas en BSR tienden a ser finalizadas por puntuaciones altas, en el baloncesto convencional éstas se dividen entre los jugadores interiores (mayor número de lanzamientos de 2 puntos cercanos al aro, rebotes y faltas recibidas) y los jugadores exteriores (mayor número de asistencias y lanzamientos de 3 puntos) (Sampaio et al., 2006).

Por estos motivos, aunque la posición de pívot y Clase 4 parecen compartir semejanzas en algunas variables de la estadística de juego (lanzamientos de $2 \mathrm{p}$, rebotes y faltas recibidas) entre el baloncesto convencional y el BSR (Gómez y Lorenzo, 2007; Gómez et al., 2014; Sampaio et al., 2006; Vanlandewijck et al., 2011), las diferencias existentes con las demás posiciones de juego y clases funcionales hacen que el baloncesto en silla de ruedas deba ser analizado y estudiado desde su propia dinámica de rendimiento (Molik et al., 2009) y sea tenida muy en cuenta el rendimiento del jugador atendiendo a su clase funcional (Crespo-Ruiz et al., 2011; Vanlandewijck et al., 2004).

Ante estas reflexiones, parece necesario establecer objetivos de rendimiento para los jugadores acorde a su clasificación funcional y su posición de juego, sirviendo los valores máximos registrados en la estadística de juego como valores de referente del rendimiento óptimo de cada jugador en cada variable para su clasificación funcional. Así mismo, la incorporación en el análisis de la estadística de juego de acciones fundamentales en el trabajo en equipo en BSR (Dávila, 2010; Gordon, 2010) tales como bloqueos, pantallas, man - outs, missmatchs, defensas individuales y ayudas defensivas puede reforzar en mayor medida la visualización y cuantificación del rendimiento de los jugadores con menor clase funcional.

\section{Aplicaciones prácticas}

El análisis descriptivo del rendimiento del jugador de baloncesto en silla de ruedas de alta competición según la clase funcional y la identificación de los rendimientos óptimos para cada clase funcional a través de la estadística de juego permiten a los técnicos y futuros entrenadores disponer de un patrón de rendimiento basado en la propia competición (Gómez et al., 2014; Nadori, 1993). Estos datos permiten además adecuar las necesidades de rendimiento a cada clase funcional, aspecto que aunque había sido estudiado desde el marco de la biomecánica y el rendimiento físico apenas ha sido estudiado durante la competición hasta el momento. Finalmente, estos datos han servido de referencia en el proceso de seguimiento y selección de los jugadores componentes de la Selección Espańola de Baloncesto en Silla de Ruedas que participaron en el pasado Campeonato del Mundo celebrado en Incheon (Korea) en Julio de 2014, logrando la $4^{a}$ posición, siendo la mejor posición lograda en la historia de esta selección, igualando la plaza obtenida en Atlanta 1996.

\section{Agradecimientos}

A la Federación Española de Deportes para Personas con Discapacidad Física (FEDDF), por facilitar el acceso a los datos estadísticos.

\section{Referencias}

1. Crespo-Ruiz, B. M., Del Ama-Espinosa, A. J., \& Gil-Agudo, Á. M. (2011). Relation Between Kinematic Analysis of Wheelchair Propulsion and Wheelchair Functional Basketball Classification. Adapted Physical Activity Quarterly, 28(2), 157-172.

2. Dávila, A. (2010). Manual para el entrenador del baloncesto sobre silla de ruedas. México: CONADE.
3. De Groot, S., Balvers, I. J. M., Kouwenhoven, S. M., \& Janssen, T. W. J. (2012). Validity and reliability of tests determining performancerelated components of wheelchair basketball. Journal of Sports Sciences, 30(9), 879-887.

4. de Lira, C. A. B., Vancini, R. L., Minozzo, F. C., Sousa, B. S., Dubas, J. P., Andrade, M. S., . . . da Silva, A. C. (2010). Relationship bet- 
ween aerobic and anaerobic parameters and functional classification in wheelchair basketball players. Scandinavian Journal of Medicine \& Science in Sports, 20(4), 638-643.

5. FIBA. (2005). Basketball statistics manual. FIBA assist magazine(15), 40-44.

6. Gómez, M. A., \& Lorenzo, A. (2007). Análisis discriminante de las estadísticas de juego entre bases, aleros y pivots en baloncesto masculino. Apunts. Educación Física y Deportes, 16(1), 41-54.

7. Gomez, M. A., Lorenzo, A., Sampaio, J., Ibanez, S. J., \& Ortega, E. (2008). Game-related statistics that discriminated winning and losing teams from the Spanish men's professional basketball teams. Coll Antropol, 32(2), 451-456.

8. Gómez, M. A., Pérez, J., Molik, B., Szyman, R. J., \& Sampaio, J. (2014). Performance analysis of elite men's and women's wheelchair basketball teams. Journal of Sport Sciences. doi: 10.1080/02640414.2013.879334

9. Gordon, T. (2010). Wheelchair basketball leaders award and education pack. Great Britain: GBWBA.

10. IWBF. (2010). Official player classification manual. Canada: IWBF

11. Leicht, C. A., Bishop, N. C., \& Goosey-Tolfrey, V. L. (2012). Submaximal exercise responses in tetraplegic, paraplegic and non spinal cord injured elite wheelchair athletes. Scandinavian Journal of Medicine \& Science in Sports, 22(6), 729-736.

12. Mason, B., Woude, L., \& Goosey-Tolfrey, V. (2013). The Ergonomics of Wheelchair Configuration for Optimal Performance in the Wheelchair Court Sports. Sports Medicine, 43(1), 23-38.

13. Molik, B., Kosmol, A., Morgulec'Adamowicz, N., Laskin, J. J., Jezior, T., \& Patrzałek, M. (2009). Game efficiency of elite female wheelchair basketball players during world championships (Gold cup) European Journal of Adapted Physical Activity, 2(2), 26-38.

14. Molik, B., Kosmol, A., Morgulec, N., Hübner-Woźniak, E., \& Rutkowska, I. (2006). Anaerobic Performance in Polish First League Team of Wheelchair Basketball Players. Research Yearbook, 12(2), 199-202.

15. Nadori. (1993). El talento y su selección. Algunos problemas teóricos y metodológicos de la selección del talento deportivo. Rivista di Cultura Sportiva, 28-29, 101-108.
16. Pérez, J. (2003). Análisis del perfil de exigencia en el baloncesto en silla de ruedas. . Universidad Politécnica de Madrid.

17. Pérez, J. (2012). Baloncesto en silla de ruedas. En Deportistas sin adjetivos: el libro de la FEDDF. Federación Española de Deportes para Personas con Discapacidad Física, CSD, CPE: Madrid. Págs 303 - 353.

18. Pérez, J., \& Sampedro, J. (2003). Progresión para la iniciación al baloncesto en silla de ruedas. Revista Digital, 8(57).

19. Sampaio, J., Janeira, M. A., Ibanez, S. J., \& Lorenzo, A. (2006). Discriminant analysis of game-related statistics between basketball guards, forwards and centres in three professional leagues. European Journal of Sport Science, 6(3)

20. Sampaio, J., Lorenzo, A., Gómez, M. A., Malarranha, J. M., Ibáñez, S. J., \& Ortega, E. (2009). Análisis de las estadísticas discriminantes en jugadores de baloncesto según su puesto específico, en las finales de las competiciones europeas (1988-2006). Apunts. Educación Física y Deportes, 96, 53-58.

21. Skučas, K., Stonkus, S., Molik, B., \& Skučas, V. (2009). Evaluation of wheelchair basketball skill performance of wheelchair basketball players in different game positions. Education. Physical Training. Sport (75), 65-69

22. Tweedy, S. M., \& Vanlandewijck, Y. C. (2011). International Paralympic Committee position stand-background and scientific principles of classification in Paralympic sport. British Journal of Sports Medicine, 45(4), 259-269.

23. Vanlandewijck, Y. C., Evaggelinou, C., Daly, D. J., Verellen, J., Houtte, S. V., Aspeslagh, V., . . . Zwakhoven, B. (2004). The relationship between functional potential and field performance in elite female wheelchair basketball players. Journal of Sports Sciences, 22(7), 668675.

24. Vanlandewijck, Y. C., Verellen, J., \& Tweedy, S. (2011). Towards evidence-based classification in wheelchair sports: Impact of seating position on wheelchair acceleration. Journal of Sports Sciences, 29(10), 1089-1096. 